\title{
SUBJETIVIDAD Y MATERIALIDAD DEL CUIDADO: ÉTICA, TRABAJO Y PROYECTO POLÍTICO
}

Subjectivity and materiality of care: ethics, work and political project

Pascale Molinier*; Matxalen Legarreta**
*UTRPP EA 4403, Université Paris 13 SPC; ${ }^{* *}$ Universidad del País Vasco/Euskal Herriko
Unibertsitatea
pascale.molinier@cnam.fr; matxalen.legarreta@ehu.es

Molinier, P., Legarreta, M., 2016, "Subjetividad y materialidad del cuidado: ética, trabajo y proyecto político", en Papeles del CEIC, vol. 2016/1, presentación, CEIC (Centro de Estudios sobre la Identidad Colectiva), Universidad del País Vasco, http://dx.doi.org/10.1387/pceic.16084

\section{SUBJETIVIDAD Y MATERIALIDAD DEL CUIDADO}

En francés el término más cercano a cuidado es soin. Soin incluye tanto la dimensión curativa del cuidado como su sentido de atención o de preocupación, sin que ambos puedan distinguirse conceptualmente. Por ello, en Francia, las investigadoras feministas que trabajan sobre el cuidado han optado por no traducir el término inglés care para incorporarlo en su lengua en cursiva y sin comillas (Paperman y Laugier, 2005). De esta forma, a través de la noción care se identifica y se hace explícita la dimensión no médica de la atención y de la preocupación.

El término cuidado en español, por su parte, deriva del latín cogitatus. Cogitatus es un nombre que deviene del participio cogitare (que significa pensar, reflexionar) y que hace referencia a la reflexión, el pensamiento, el interés reflexivo que una persona pone en algo. El verbo cogitare se compone de co- (acción conjunta o global) y agitare (poner en movimiento, agitar, darle vueltas a las cosas), un frecuentativo de agere, llevar adelante, hacer avanzar, mover, tratar, actuar. Etimológicamente, por tanto, el cuidado en español recoge las dos dimensiones que engloba el verbo en inglés, la subjetiva -el cuidado como preocupación, responsabilidad, disposición (care about) - y la material -el cuidado como acción, ocupación (care for)-. Además, cuidar se diferencia de curar, que posee una acepción más médica. De tal modo, en el contexto español y de América Latina se ha mantenido la palabra cuidado en su idioma original tanto en la academia como en el movimiento feminista. 
Al centrarse en la dimensión no médica o curativa del cuidado y al pensarlo como un proceso colectivo ("acción conjunta o global") se pone el foco de atención más allá del personal médico o paramédico cualificado. Así, es posible trascender el ámbito concreto de la enfermedad para abordar el amplio campo de la vida cotidiana, donde todas las personas necesitamos que se ocupen de nosotras $y$, al mismo tiempo, tenemos la capacidad de ocuparnos de otras. De hecho, como sugiere su raíz etimológica, en el cuidado preocuparse por alguien significa hacer algo concreto por esa persona. Dicho de otro modo, el cuidado se aplica tanto a un trabajo tangible como a la actitud moral que se desarrolla principalmente a través de, y gracias a, dicho trabajo. Preocuparse por el cuidado implica, por tanto, considerar sus condiciones organizativas y materiales, es decir, ocuparse, lo que específicamente conlleva una división del trabajo.

No obstante, la división del trabajo de cuidado tiene unas características particulares. Está distribuido de manera desigual en nuestras sociedades: recae principalmente sobre las mujeres y está atravesada también por desigualdades de raza y de clase. Es un trabajo, además, que ha sido relegado a la esfera de lo privado, a lo doméstico y al hogar. Por consiguiente, las voces de las personas proveedoras de cuidados apenas han sido escuchadas y han tenido menor consideración que las de otros actores con mayor protagonismo en la esfera pública. Por todo ello, desde el feminismo, al poner el foco de atención en el cuidado se pretende poner de manifiesto las desigualdades que caracterizan su reparto.

Partiendo de estas premisas, ubicarse desde la perspectiva del cuidado supone dar a estas actividades, así como a la ética consustancialmente incorporada a ellas, un valor propio, que no se subordina ni a las éticas médicas, ni a las éticas clásicas de la justicia. De tal forma, se reconoce la existencia de un trabajo concreto y de una ética específica, la que surge de las personas subalternas, representantes privilegiadas de lo que la psicóloga Carol Gilligan (1982) ha identificado como una "voz diferente".

\section{LA ÉTICA DEL CUIDADO}

La ética del cuidado -ethics of care - nace en Estados Unidos con la obra de Carol Gilligan (1982) en el ámbito de la psicología moral y se desarrolla en el campo de los estudios feministas. En su libro In a different voice ${ }^{1}$

\footnotetext{
${ }^{1}$ De este texto, del que citamos su edición en inglés (Gilligan, 1982), hay traducción al castellano: Gilligan, C., 1985, La moral y la teoría. Psicología del desarrollo femenino, Fondo
} 
Gilligan (1982) pone en evidencia la importancia de una forma de razonamiento moral considerada hasta el momento "menos" moral, porque no responde a los criterios de abstracción y de generalización que definen la "ética de la justicia". Ante el conocido dilema de Heinz utilizado en la psicología moral, dos criaturas de 11 años, Jack y Amy, ofrecen a Gilligan una respuesta diferenciada. El dilema es el siguiente:

Heinz vive en un país extranjero con su esposa enferma. El farmacéutico tiene una medicina que puede salvarla, sin la cual la esposa morirá. Heinz no tiene el dinero para comprarla y el farmacéutico se niega a dársela. ¿Debe Heinz robar la medicina?

Jack responde que sí: Heinz debería robar la medicina, pues la vida vale más que la propiedad y el juez que lo juzgue por el robo lo entenderá. La respuesta de Amy no coincide con la de Jack: "Bueno, yo no lo creo, dice ella. Puede haber otras maneras de salir adelante sin tener que robar la medicina: podría pedir dinero prestado, por ejemplo. Pero en realidad no debería robar ni tampoco su esposa debería morir" (Gilligan, 1982: 50). Amy señala que, si Heinz va a la cárcel por el robo, su esposa se quedará desvalida si vuelve a enfermar. Ella sugiere que Heinz y su esposa deberían hablar entre ellos y encontrar la forma de recaudar fondos o de convencer al farmacéutico para que les diera la medicina.

Ante el mismo dilema, Jack responde según los criterios de la ética de la justicia. Amy, sin embargo, lo convierte en un problema de responsabilidad relacional: es el farmacéutico quien tiene la responsabilidad moral y Heinz no puede decidir en lugar de su esposa. A través de su razonamiento, Amy no busca un arbitraje superior (el juez), sino que invita a las personas protagonistas del relato a buscar juntas una solución que no está predeterminada. Ante esta situación Gilligan recapitula:

"En este concepto [ética del cuidado], el problema moral surge de las responsabilidades que chocan entre sí y no de una competencia entre derechos y requiere para su resolución una modalidad de pensamiento contextual y narrativa, en vez de una formal y abstracta. Este concepto de moralidad, en tanto que muestra preocupación por la actividad del cuidado, centra el desarrollo moral alrededor de la comprensión de la responsabilidad y de las relaciones, de la misma manera que el concepto de moralidad como equidad une el desarrollo moral

de Cultura Económica, México; y al francés: Gilligan, C., 2009, Une voix différente, Pour une éthique du cuidado, Champs essais, Paris. 
con el entendimiento de los derechos y las reglas." (Gilligan, 1982: 59).

El libro de Gilligan pone en evidencia un vínculo -cuanto menos empírico- entre las mujeres y la ética del cuidado, por lo que su trabajo ha sido a menudo entendido como una muestra de que las mujeres son diferentes a los hombres y de que la ética del cuidado es una moral femenina. No obstante, Gilligan ha afirmado que no considera la ética del cuidado como una categoría de la diferencia de género. Para ella, la ética del cuidado se puede confundir con "la moralidad de las mujeres", pero no por su supuesta "naturaleza", sino por la socialización de género que se desarrolla desde la primera infancia. De este modo, sitúa su reflexión en el horizonte del patriarcado, respondiendo a las críticas a través de la distinción entre, por un lado, una ética femenina del cuidado, la del "ángel del hogar", basada en la entrega de sí y, por otro, una ética feminista del cuidado, basada en el respeto de sí y en el reparto de las responsabilidades (Gilligan, Tronto, Hochschild, 2013; Gilligan, 2013).

\section{EL CUIDADO: TRABAJO Y MORAL SOCIAL}

La politóloga Joan Tronto realiza una contribución importante al debate sobre la ética del cuidado al subrayar que no se trata tanto de una moral femenina, sino de una moral social. Las distintas expresiones de las mujeres sobre la moralidad pueden derivar de la función que desempeñan según su posición social subordinada. Tronto defiende su postura en base a tres argumentos que, si bien desarrolla de forma más pormenorizada en su obra (Tronto, 1987), sintetiza de la siguiente manera:

\footnotetext{
“Primero, equiparar 'el cuidado' con 'la mujer' es cuestionable, porque la evidencia que apoya el vínculo entre la diferencia de género y la diferencia de la perspectiva moral es inadecuada. Segundo, es una postura estratégica peligrosa para las feministas, porque la mera declaración de la diferencia de género en un contexto social en el que se identifica al hombre como normal contiene una implicación de inferioridad para lo que pertenece únicamente a las mujeres. Tercero, filosóficamente este argumento puede anularse, porque si las feministas piensan la ética del cuidado como categoría de la diferencia de género, es muy probable que caigan en la trampa de intentar defender la moralidad de las mujeres en vez de mirar críticamente las posibilidades y los problemas filosóficos de una ética del cuidado" (Tronto, 1987: 2).
} 
En la perspectiva de Tronto la ética del cuidado es propia de las personas que se ocupan de otras personas, en particular de las más vulnerables: un trabajo a cargo mayoritariamente de las mujeres (no de todas) y de aquellos hombres que forman parte de grupos subalternos. Desde esta mirada, la ética del cuidado se basa en, y se realiza a través de, un trabajo de cuidado. Esta distinción supone un avance importante para las propuestas feministas porque abre la posibilidad teórica y política de no desligar el trabajo y la ética del cuidado. De esta forma, siguiendo la definición de Tronto, el cuidado se define como:

\begin{abstract}
"una actividad característica de la especie humana que incluye todo lo que hacemos con vistas a mantener, continuar o reparar nuestro 'mundo', de tal manera que podamos vivir en él lo mejor posible. Este mundo incluye nuestros cuerpos, nuestras individualidades (selves) y nuestro entorno, que buscamos tejer juntos en una red compleja que sostiene la vida" (Tronto, 1993: 103, citado en Paperman, 2011).
\end{abstract}

Según esta propuesta, el trabajo de cuidado es todo el trabajo que se necesita para mantener un mundo común. Disfrutar de un mundo común parece algo sencillo, lo damos por supuesto y tendemos a pensar que está siempre ahí, pero no es así. El mundo común se construye día tras día, noche tras noche, a través de un trabajo que no conoce límites en el tiempo y que se evidencia solamente cuando hay desgaste, cuando el mundo común desaparece o se desvanece. De esta forma, si bien en el pensamiento ordinario el mundo común está aquí y pensamos con seguridad que seguirá estando, no es así, puesto que es el resultado de todo un trabajo: el trabajo de cuidado.

La cuestión radica entonces en saber quién responde a nuestras necesidades ordinarias de limpieza, confort, tranquilidad... y contribuye así a preservar una forma de vida humana. La pregunta clave es: ¿quién sostiene la vida y cómo?

\title{
4. EL CUIDADO Y LAS RELACIONES DE PODER
}

Si bien emergen en Estados Unidos, actualmente las investigaciones sobre el cuidado se desarrollan en el mundo entero, y este monográfico es una muestra de ello. El desarrollo de los estudios sobre el cuidado se relaciona con el aumento y generalización del trabajo asalariado de las mujeres menos disponibles y dispuestas hoy que antaño a desempeñar gratuitamente el trabajo de cuidado-, así como con el aumento de la 
esperanza de vida y el crecimiento del número de personas mayores que requieren atención. No obstante, más ampliamente, desde la perspectiva del cuidado, la vulnerabilidad humana es genérica, por lo que se considera que todas las personas somos destinatarias de cuidado: bien criaturas, mayores y personas dependientes, bien personas adultas "competentes". De esta forma, las investigaciones en torno al cuidado llevan implícita una crítica al modelo de "autonomía plena", por considerarla parcial y, sobre todo, precaria y provisoria.

Las teorías del cuidado promueven, por tanto, un reconocimiento sin precedentes de la vulnerabilidad como una condición común de la humanidad. A este respecto, Tronto señala que:

\begin{abstract}
"hay un continuo en el grado de atención que cada persona necesita, no una dicotomía entre aquellas que son atendidas y las que las cuidan. Pero ésta no es una idea que la mayoría de las personas aceptan fácilmente. Se supone que nos consideramos seres vulnerables. Esto implica que abandonemos nuestro sentido de autonomía plena. Y requiere que dejemos de pensar que la "autonomía personal" es la solución a todos los problemas de la sociedad" (Tronto, 2009: 33).
\end{abstract}

De hecho, el reconocimiento de nuestra propia vulnerabilidad y del hecho de estar vinculados y vinculadas a las demás personas, pueden llegar a cambiar nuestra forma de pensar acerca del trabajo que es necesario llevar a cabo para sostener nuestras vidas y para construir un mundo común, así como sobre de la responsabilidad social que inevitablemente ello conlleva.

Al mismo tiempo, los estudios sobre el cuidado vuelven gradualmente más visibles las nuevas categorías de trabajadoras, en su mayoría mujeres con escasos recursos económicos, a menudo migrantes, a veces en situación ilegal $y$, al menos en los Estados español y francés, frecuentemente empleadas por debajo de su cualificación. ¿Cómo explicar que confiemos el cuidado de las personas más frágiles de nuestro entorno más cercano (hijos e hijas, padres y madres...) a mujeres a quienes pagamos menos de lo que les corresponde y a quienes, además, a menudo menospreciamos tan solo porque provienen de países económicamente más débiles y, en lo que respecta a Francia, por ejemplo, de países que son ex-colonias?

El cuidado no puede pensarse fuera de las relaciones de dominación: relaciones asimétricas entre hombres y mujeres, pero también entre clases y razas. En efecto, la relación social de dominación que define y configura el trabajo de cuidado como un trabajo subalterno se inscribe en las 
dinámicas Norte/Sur, países más ricos/países más pobres, difícilmente disociables del racismo y de los efectos prolongados del colonialismo. La colonialidad del poder pasa por las formas de "dominación cercana" (Memmi, 2003) que se establecen en el espacio doméstico: entre mujeres, en torno a los cuidados del cuerpo. Precisamente, en aquello que se considera como lo más privado y menos político.

Desde esta mirada, el cuidado deja de ser una moral de los buenos sentimientos y se convierte en un proyecto para superar las fronteras entre lo privado y lo político, entre el trabajo y la ética.

\section{Más AlLÁ de LA CULTURA DEL TRABAjO}

Hasta la década de los ochenta del siglo pasado la categoría trabajo ha englobado únicamente el empleo o la ocupación remunerada, por lo que ha excluido a las mujeres y a las actividades domésticas asignadas a ellas. La noción de trabajo emerge en relación directa con el desarrollo del capitalismo industrial y la construcción de las democracias occidentales. La preservación del lugar de trabajo como ámbito de solidaridad fraternal (entre iguales) se conserva a lo largo de todo el siglo XX, con efectos sobre el concepto trabajo (qué incluye y qué no) y sobre la categoría de "trabajadores" (quiénes lo son y quiénes no). En este marco, las actividades de cuidado se encuadran, categorizadas como no trabajo, en el ámbito privado.

En este contexto, en las ciencias del trabajo que acompañan el desarrollo de las sociedades industriales, el paradigma dominante es el del trabajo obrero, necesariamente masculino, aún cuando el trabajo estudiado sea el de las mujeres. En Francia, por ejemplo, durante la década de los sesenta del siglo pasado, la mayoría de las investigaciones fundacionales de la ergonomía se desarrollan en sectores feminizados (como por ejemplo, electrónica, confección y recepción telefónica). Sin embargo, en los procesos de generalización, la obrera se convierte, bajo la pluma de los investigadores, en un trabajador indiferenciado. Esta neutralización de las obreras que efectivamente desempeñan dichas ocupaciones bajo la figura abstracta del trabajador (asumido como masculino), deja el campo libre a los fuertes prejuicios según los cuales las mujeres están mejor adaptadas a las ocupaciones "rutinarias y fáciles", al trabajo repetitivo y regular, al compás de la supuesta monotonía y simplicidad de las tareas domésticas. 
Ante esta situación, la segunda ola del feminismo visibiliza el trabajo femenino, tanto asalariado como doméstico, y le da valor. Así, algunas sociólogas proponen un concepto de trabajo más amplio o extenso que incluye el trabajo doméstico y la crianza de las criaturas, al destacar que forman pare de los procesos de "producción de la vida" (Kergoat, 2011). Otras llegan incluso a considerar la sexualidad conyugal como parte del trabajo reproductivo de las mujeres (Tabet, 1985). Las actividades asignadas prioritariamente a las mujeres adquieren así (al menos en el plano teórico) la dignidad que confiere la categoría trabajo, al tiempo que se desarticula su definición (Kergoat, 2011), y su vinculación con el trabajo remunerado (mercado laboral).

\section{LA DISTRIBUCIÓN DELTRABAJO DE CUIDADO}

Una parte de lo que hasta ese momento se confunde con amor conyugal, amor filial, amor por los hijos y las hijas, adquiere la consideración de trabajo (no remunerado, no reconocido como parte de la riqueza del país $y$, sin embargo, indispensable para la sostenibilidad de la vida). A partir de este giro, se abre la posibilidad de considerar el reparto del trabajo de cuidado entre los miembros de la pareja, así como a nivel social. No obstante, dicho reparto no siempre se lleva a cabo de manera satisfactoria. Así, el fracaso de la división conyugal de las tareas, junto con una provisión de servicios de cuidado insuficiente por parte de las instituciones, conlleva la división del trabajo de cuidado entre las mujeres a través de la externalización, a menudo, de las tareas más rutinarias y menos placenteras. De este modo, ante la ausencia de una transformación significativa respecto de la "organización social del cuidado" (Daly y Lewis, 2011), el mito glorificador del trabajo como promesa de felicidad, de logro moral, e incluso de salud mental (Molinier, 2013), continua edificándose sobre la base de la negación o del ocultamiento de aquello que el trabajador eficiente del neoliberalismo, el "trabajador champiñón" (Pérez Orozco, 2010), le debe al trabajo doméstico realizado para su beneficio.

Además, la "cultura del trabajo" establece un modelo de sociedad donde la división del trabajo juega un rol central en la atribución de las riquezas, en la jerarquización social y en el reconocimiento de las personas. Ahora bien, la división del trabajo se vincula estrechamente con la idea de especialización -asociada generalmente a la puesta en práctica de técnicas, monopolio masculino por excelencia (Tabet, 1998) - y ésta se considera una garantía de eficacia en el trabajo. Sin embargo, la 
descualificación de las especialidades no técnicas y no especializadas resulta desproporcionada y repercute directamente en el trabajo de cuidado remunerado, así como en su reconocimiento como fundamento de la civilización.

Todo ello explica, en parte, por qué el trabajo de cuidado, dirigido a satisfacer las necesidades de las personas y a construir un mundo común, forma parte de las profesiones con menor remuneración y menos respetadas. Se trata, en efecto, de un trabajo feminizado, a menudo invisible, en parte realizado gratuitamente en el ámbito privado donde se confunde con el amor y la solidaridad, frecuentemente confiado a migrantes estigmatizadas... En suma, un trabajo no técnico ni especializado. En otras palabras, el cuidado es un trabajo, pero no tiene nada de espectacular, pues forma parte inherente de la vida misma. Esto es, permite a la vida cotidiana mantenerse, con lo que hace posible la pervivencia de las personas, pues los seres humanos necesitamos inexorablemente una rutina (repetición) con sentido y con puntos de referencia familiares. El cuidado, por tanto, sostiene la vida.

\section{LA MEDICIÓN DEL CUIDADO Y SUS LÍMITES}

El trabajo de cuidado corre el riesgo de ser descalificado, entre otros, por la dificultad que conlleva cuantificar su ejecución y, más aún, su eficacia. No obstante, los esfuerzos en torno a la cuantificación han sido importantes para las investigaciones feministas. Dicha labor se ha realizado principalmente a través de las Encuestas de Empleo de Tiempo y de las Cuentas Satélite de Producción Doméstica. La medición cuantitativa del volumen de trabajo llevado a cabo en los hogares ha permitido poner de manifiesto su contribución al bienestar y a la riqueza de los países, estimar su peso relativo respecto al trabajo productivo-mercantil, hacer visible la magnitud de su reparto desigual y sacar a la luz la contribución de sectores de la población identificados a menudo como dependientes (población mayor, por ejemplo). En definitiva, produce datos sobre la materialidad del cuidado, pero no sobre su subjetividad.

Esta contribución de las estimaciones cuantitativas del cuidado está, sin embargo, estrechamente ligada a la aceptación y al reconocimiento de sus limitaciones. La más elemental es que la lógica que subyace a la cuantificación es la lógica productivista-mercantil, que invisibiliza e infravalora el cuidado (Legarreta, 2012). Las Encuestas de Empleo de 
Tiempo calculan el volumen de tiempo que se invierte en las ocupaciones de cuidado mediante una noción de tiempo cuantitativa, abstracta y descontextualizada - horas y minutos-, que surge y se generaliza junto la concepción moderna de trabajo y que lleva implícitos los valores de eficacia y rapidez ("el tiempo es oro"). Las Cuentas Satélite de Producción Doméstica, por su parte, estiman el coste que supone realizar el trabajo de cuidado a través del mercado tomando en cuenta el salario por hora de una persona que trabaja de forma polivalente desempeñando diversas tareas del hogar (limpieza, atención, gestión...), en un contexto en el que los salarios se estipulan con base en la especialización y la cualificación y en el que los ingresos son menores para los sectores feminizados.

Se puede contar el número de camisas planchadas, las horas y minutos que requiere el planchado, pero ¿cómo se mide una sonrisa?, ¿y el cariño (o el hastío) con el que se cambia un pañal? El cuidado requiere un modo de relación particular que no es fácil cuantificar: responde a otra gramática, no de la medida, sino de la incertidumbre narrativa. Entonces, para entender las maneras en las que se organiza el trabajo de cuidado y las formas en las que se da respuesta a las necesidades de las demás personas, más allá de la cuantificación, hay que pensar también otras formas de producción de datos - tales como los relatos, las historias y las narraciones contextuales-, que permitan acceder al sentido y a las representaciones del cuidado.

La revisión de las herramientas analíticas y la manifestación de su alcance resulta fundamental para tratar el cuidado. Por ello, la perspectiva del cuidado implica una crítica a los instrumentos de medida del neoliberalismo. Esto es, significa una inversión tanto en el orden de los saberes como en el de las prioridades humanas $y$, por tanto, políticas.

\section{ESTRUCTURA DEL MONOGRÁFICO}

El monográfico aborda el cuidado a partir de los tres ejes desarrollados en los epígrafes anteriores: trabajo, ética y proyecto político. Se compone de ocho artículos escritos por académicas de diferentes lugares de Europa (Estados español y francés) y de América Latina (Argentina y Uruguay). Con ello, ofrece una panorámica de las investigaciones que se están llevando a cabo en este campo, no sólo en el contexto más cercano de las editoras sino también al otro lado del océano. Si bien se han desarrollado en diferentes entornos y han tratado diferentes tópicos, las inquietudes que 
motivan los trabajos presentados, así como sus formas de abordar el estudio del cuidado, coinciden en varios sentidos y no es difícil detectar sinergias y puntos de encuentro entre los artículos. Estas sinergias y puntos de encuentro se extienden también a las reseñas de libros y las críticas audiovisuales que completan el número monográfico.

Los primeros cuatro artículos centran la atención en la provisión de cuidados, a través de investigaciones cualitativas, poniendo el foco en los discursos de las personas cuidadoras, sus idearios y su organización del trabajo. El texto de Sara Moreno, Carolina Recio, Vicent Borràs y Teresa Torns (Universidad Autónoma de Barcelona) da cuenta de un estudio llevada a cabo en Barcelona para analizar las representaciones de las cuidadoras formales e informales sobre el "ideal de cuidados", esto es, los significados e imaginarios sociales que condicionan las preferencias sobre cómo organizar los cuidados de larga duración. El artículo de Paloma Moré (Universidad Complutense de Madrid) centra la atención en las residencias para personas mayores a través de una investigación realizada en Madrid y Paris con el fin de estudiar los efectos que tiene la organización social del trabajo de cuidados sobre quienes lo llevan a cabo y problematizar, de este modo, la relación de los cuidados con el universo inmaterial (afectos, emociones y amor). Mira Younes y Pascale Molinier (Universidad Paris 13) muestran un trabajo etnográfico centrado en las trabajadoras domésticas en Colombia y Líbano para abordar las relaciones de dominación que atraviesan la familia como "institución política naturalizada" e invitar a una lectura política del lenguaje de las relaciones familiares y/o afectivas en el marco de la interacción entre las empleadas y las personas empleadoras. La mirada de Natacha Borgeaud-Garciandía (FLACSO-CONICET) reposa también en las trabajadoras domésticas, en este caso situadas en Argentina, y pone de manifiesto las estrategias desarrolladas por ellas con el fin de proteger y resignificar su trabajo en contextos que les suponen una confrontación con elementos perturbadores y potencialmente desestabilizantes como la intimidad, la sexualidad y la demencia senil de adultos mayores muy dependientes.

Los cuatro siguientes trabajos versan sobre aspectos más diversos y no todos son de corte cualitativo. Laura Marzi y Patricia Paperman (Universidad Paris 8) plantean un acercamiento transdisciplinar entre la sociología y la literatura que pretende restaurar la importancia ética de las relaciones de cuidado que se desarrollan en el ámbito doméstico a través del estudio de las formas en las que la vida cotidiana se hace visible y 
reconocible en los textos literarios. Rosario Aguirre y Sol Scavino (UdelaR) abordan las desigualdades de género en la provisión de cuidados por parte de las personas mayores a través del análisis los datos cuantitativos de las Encuestas de Uso del Tiempo de Uruguay, sacando a la luz su contribución al bienestar social y familiar y no sólo como dependientes. Marina Sagastizabal y Matxalen Legarreta (Universidad del País Vasco, UPV/EHU) presentan una propuesta de corte analítico que trata tanto la materialidad como la subjetividad del cuidado, a través de la noción "triple presenciaausencia", partiendo de una mirada amplia que engloba la relación del cuidado con el trabajo remunerado y con la participación sociopolítica. Para finalizar, el trabajo cualitativo de Amaia Agirre (Universidad del País Vasco UPV/EHU) llevado a cabo en la Comunidad Autónoma del País Vasco, estudia los procesos de negociación desarrollados en las parejas que se autodefinen como paritarias en torno a tres ámbitos, que según pone de manifiesto, tienen una consideración diferente entre las parejas: los trabajos domésticos, la crianza y la construcción de la maternidad y la paternidad.

El número se completa con tres reseñas de libros y dos reseñas audiovisuales. Carmen Crespo (Universidad del País Vasco, UPV/EHU) nos ofrece la del libro Subversión feminista de la economía. Aportes para un debate sobre el conflicto capital-vida de Amaia Pérez Orozco en el que, desde la centralidad del cuidado, la economista feminista establece las bases de su propuesta para analizar lo que llama "el conflicto capital-vida". Siguiendo la línea argumental de sus anteriores trabajos, Orozco propone una lectura alternativa del sistema económico utilizando, para ello, herramientas que escapan a los análisis hegemónicos y ampliando la mirada con el fin de desplazar el foco hacia preguntas que cuestionan el status quo: ¿por qué? y ¿para qué sostener dicho sistema?, ¿acaso conduce a vidas que merezcan la pena ser vividas? Dornaku Lanz (Universidad del País Vasco, UPV/EHU) presenta la obra editada por María José González y Teresa Jurado Padres y madres corresponsables. Una utopía real, que da cuenta de una investigación cualitativa longitudinal llevada a cabo en varias ciudades del contexto español con parejas antes y después de tener descendencia. El trabajo estudia las decisiones tomadas respecto al empleo y al trabajo de cuidados durante el embarazo y los primeros meses de crianza del bebé. A partir de las reflexiones que se derivan de su análisis ponen encima de la mesa las posibilidades que plantea una forma igualitaria de parentalidad, esto es, una "utopía real" de la 
corresponsabilidad. Julie Aguirre (Universidad del País Vasco, UPV/EHU) reflexiona sobre el texto Studies in global social history. Black girls migrant domestic workers and colonial legacies de Sabrina Marchetti. En él se exploran, desde una óptica interseccional, las maneras en las que el legado colonial ha afectado a la formación de la identidad de los sujetos migrantes y su relación directa con el nicho de los cuidados y del trabajo doméstico. El libro da cuenta de una investigación comparativa que aborda los casos de trabajadoras domésticas migrantes post coloniales que, procedentes de Eritrea y de Surinam, viven en Italia y en Holanda respectivamente.

Oihana Garro (Universidad del País Vasco, UPV/EHU) desarrolla una crítica audiovisual sobre la película Amour de Michael Haneke. Rodado integramente el en interior de una casa y protagonizada por una pareja de personas ancianas, el film sirve como vehículo para presentar una reflexión sobre el cuidado a partir de tres aspectos clave: dicotomía público/privado, cuidado como trabajo y vulnerabilidad como dimensión constituyente de lo humano. Por último, Aintzane Rincón (Universidad del País Vasco, UPV/EHU) analiza la película Loreak dirigida por Jon Garaño y Jose Mari Goenaga, cuyo eje argumental gira en torno a unos ramos de flores que unen en un mismo hilo narrativo a tres mujeres que aparentemente no tienen vínculos entre sí. Centrando la mirada en las tres protagonistas, se desarrolla una reflexión que pone el acento en la construcción de la feminidad en tanto preservadora de la memoria y sujeto que procura el cuidado.

\section{BibLiografía}

Daly, M., Lewis, J., 2011, “El concepto de 'social care' y el en análisis de los estados de bienestar contemporáneos", en C. Carrasco, C. Borderías y T. Torns (Eds.), El trabajo de cuidados. Historia, teoría y políticas, Catarata, Madrid, pp. 225-251.

Gilligan, C., 1982, In a different voice. Psychological theory and womens's development, Harvard University Press, Cambridge.

Gilligan, C., 2013, La Ética Del Cuidado. Carol Gilligan. Cuadernos de la Fundació Víctor Grifols i Lucas 30, Fundació Víctor Grífols i Lucas, Barcelona. 
Gilligan, C., Hochschild, A., Tronto, J., 2013, Contre l'indifférence des privilégiés. À quoi sert le care (editado y presentado por Patricia Paperman y Pascale Molinier), Payot, París.

Kergoat, D., 2011, Se battre, disent-elles, La Dispute, Paris.

Legarreta, M., 2012, El tiempo donado en el ámbito doméstico-familiar. Estudio sobre el trabajo doméstico y de cuidados. Tesis Doctoral Inédita. Universidad del País Vasco, Leioa. Disponible en: https://addi.ehu.es/bitstream/10810/11353/1/TesisMATXALEN\%20L EGARRETA\%20IZA.pdf. Última consulta: 20/02/2016.

Memmi, D., 2003, "Une situation sans issue? Le difficile face à face entre maitres et domestiques dans le cinéma anglais et français", en Cahiers du Genre, 35, pp. 209-235.

Molinier, P., 2013, Le travail du care, La Dispute, Paris.

Paperman, P. (2011), "La perspectiva del care: de la ética a lo político", en L. G. Arango y P. Molinier (Eds.), El Trabajo y la ética del cuidado, La Carreta Social, Medellin, pp. 25-44.

Paperman, P., Laugier, S. (Eds.), 2005, Le Souci des Autres: Éthique et politique du care, vol. 16, Éditions de l'École des Hautes Études en Sciences Sociales, Paris.

Pérez Orozco, A., 2010, "Diagnóstico de la crisis y respuestas desde la economía feminista", en Revista de Economía Crítica, 9, pp. 131-144.

Tabet, P., 1985, "Fertilité naturelle, reproduction forcée", en N. ClaudeMathieu (Comp.), L'arraisonnement des femmes. Essai en anthropologie des sexes, Éditions de l'École des hautes études en sciences sociales, Paris, pp. 61-146.

Tabet, P., 1998, La construction sociale de l'inégalité des sexes. Des outils et des corps, L'Harmattan, Paris.

Tronto, J., 1987, "Más allá de la diferencia de género. Hacia una teoría del cuidado", en Signs: Journal of Women in Culture and Society, vol. 12, pp. 1-17 [Traducción del Programa de Democratización de las Relaciones Sociales. Escuela de Posgrado. Universidad Nacional de General San Martín. Aprobada y autorizada su publicación por la autora].

Tronto, J., 2009, "Care démocratique et démocraties du care", en P. Molinier, S. Laugier y P. Paperman (Dirs.), Qu'est-ce que le care? Souci des autres, sensibilité, responsabilité, Payot, Paris, pp. 35-55. 